\title{
Prevention of hypophosphatemia during continuous renal replacement therapy-An overlooked problem
}

\begin{abstract}
Hypophosphatemia is a common and potentially serious complication occurring during continuous renal replacement therapy (CRRT). Phosphate supplementation is required in the vast majority of patients undergoing CRRT, particularly beyond the first 48 hours. Supplementation can be provided either as a standalone oral or parenteral treatment or as an additive to CRRT solutions. Each approach has advantages and disadvantages, and clinicians must weigh the individual factors most relevant in their practice setting. Currently there are no consensus protocols for phosphate replacement in CRRT, and many centers replete phosphate in response to hypophosphatemia as opposed to pre-emptively. Repletion protocols have also been challenged in recent years by shortages in injectable phosphate solutions. More recently a commercially available phosphate-containing CRRT solution was approved in the United States, but there has been limited clinical experience with this product. In this review, we present recommendations for phosphate repletion in CRRT to prevent hypophosphatemia, and describe our experience using phosphate-containing CRRT solutions.
\end{abstract}

\section{1 | INTRODUCTION}

Continuous renal replacement therapy (CRRT) has emerged as the recommended dialysis modality for critically ill patients with severe renal failure, particularly those with hemodynamic instability. ${ }^{1}$ Compared to standard intermittent hemodialysis (IHD) therapy, CRRT provides greater overall solute clearance and fluid balance control. ${ }^{2}$ While IHD clearance achieves rapid correction of biochemical abnormalities with subsequent accumulation between treatments, CRRT aims to provide a more stable clearance that approaches the physiologic state while maintaining fluid balance.

Patients undergoing IHD, in either the acute or chronic setting, frequently have persistent hyperphosphatemia and require dietary phosphate-binders to reduce serum phosphate levels. In contrast, CRRT is associated with a risk of developing hypophosphatemia. Recent studies have linked CRRT-related hypophosphatemia to adverse outcomes in critically ill patients, including prolonged mechanical ventilation requirements, longer hospital length of stay, and mortality. ${ }^{3-5}$ The approach to managing this complication varies widely among programs, with most centers providing exogenous supplementation while others pre-emptively add phosphate to existing CRRT solutions. Both of these practices have been jeopardized in recent years due to shortages of intravenous phosphate solutions. ${ }^{6}$ More recently, a commercially available phosphate-containing CRRT solution has become available in the United States.

In this article, we will discuss the physiology of phosphate balance during renal replacement therapies and review the literature on hypophosphatemia in CRRT patients. We will present the advantages and disadvantages of different phosphate repletion strategies in patients undergoing CRRT, and provide recommendations for repletion strategy. We will also describe our single-center experiences using phosphate-added CRRT solutions, and discuss the potential role of newly available commercial phosphate-containing solutions.

\section{2 | PHOSPHATE PHYSIOLOGY}

In biologic systems, phosphorus exists in both organic (e.g. in phospholipids) and inorganic (e.g. ionized) forms. Inorganic phosphate plays a key role in many critical biological processes. For example, phosphorylation and dephosphorylation are integral steps in cellular energy storage and usage through the conversion between adenosine diphosphate (ADP) and adenosine triphosphate (ATP).

Bone, in the form of hydroxyapatite, is the largest reservoir of phosphate in the body. Outside of bone, most phosphate is stored intracellularly and, similar to potassium, serum levels may not accurately reflect available stores. Under normal physiologic conditions, dietary phosphate is readily absorbed in the small intestine, filtered at the glomerulus, and reabsorbed in the proximal tubule. The degree of tubular reabsorption is the key factor in maintaining serum levels, and is regulated by parathyroid hormone (PTH) and fibroblast growth factor 23 (FGF-23). ${ }^{7}$ With mild renal impairment, phosphate filtration decreases but a compensatory decrease in tubular reabsorption can maintain normal levels. In advanced renal insufficiency (acute or chronic), phosphate clearance becomes impaired enough to result in overt hyperphosphatemia. ${ }^{8}$ In patients with end stage renal disease (ESRD), hyperphosphatemia is an independent risk factor for mortality. ${ }^{9}$

Consequently, removal of phosphate is one important goal for dialysis therapy in patients with ESRD. Adequacy of dialysis clearance has traditionally focused on urea kinetics, but this approach may not apply to molecules with different characteristics. ${ }^{10}$ In the case of phosphate, a rate limiting step to dialytic clearance is the slow equilibration between the intracellular and extracellular compartments. Indeed, rebound increases in phosphate levels are typically seen following a standard IHD treatment, and improved 
phosphate control has been described with alternative dialysis regimens such as nocturnal or daily dialysis. ${ }^{11,12}$ Given these limitations, for patients on standard IHD (e.g. thrice weekly schedule) a mainstay of hyperphosphatemia management is the use of oral phosphate binders to decrease gastrointestinal absorption because thrice weekly IHD alone usually cannot clear sufficient phosphate to maintain target serum levels.

\section{3 | RATIONALE FOR PHOSPHATE REPLETION IN CRRT}

In contrast to IHD, the continuous nature of CRRT avoids any rebound effect and allows for constant phosphate clearance as compartmental equilibration occurs. ${ }^{13}$ However, dialysis solutions (commercially available dialysate and intravenous ultrafiltrate replacement solutions) traditionally have not contained any phosphate, probably because they were originally based on the electrolyte needs of ESRD patients. Therefore, unlike IHD, the primary phosphate disturbance complicating CRRT is hypophosphatemia.

In their single-center experience, Demirjian and colleagues observed a $27 \%$ incidence of hypophosphatemia (serum phosphate $<2 \mathrm{mg} / \mathrm{dL}$ ) during CRRT. ${ }^{3}$ Hypophosphatemia was associated with higher risk for prolonged respiratory failure needing tracheostomy, but there were no differences in mortality at 28 days. The association between hypophosphatemia and prolonged ventilator requirements has also been observed in critically ill patients not requiring dialysis. ${ }^{14,15}$ More recently, Yang and colleagues analyzed a cohort of 760 patients undergoing CRRT and observed hypophosphatemia $(<2.5 \mathrm{mg} / \mathrm{dL})$ in $69 \%$, and severe hypophosphatemia $(<1.0 \mathrm{mg} / \mathrm{dL})$ in $14 \%{ }^{4}$ Patients with a higher proportion of CRRT treatment days complicated by hypophosphatemia had greater mortality. In a secondary analysis of the RENAL randomized clinical trial, Bellomo and colleagues reported that $32 \%$ of patients undergoing CRRT developed hypophosphatemia ( $<1.88 \mathrm{mg} / \mathrm{dL}$ ), and hypophosphatemia was more likely with higher doses of CRRT. ${ }^{16}$ They also observed that peak incidence of hypophosphatemia occurred on day 3 or 4 of CRRT.

Thus, hypophosphatemia commonly complicates CRRT and appears to be associated with adverse clinical outcomes. Notably, hypophosphatemia frequently developed in the setting of exogenous supplementation (either enteral or parenteral), and despite many patients starting out with hyperphosphatemia. In order to avoid hypophosphatemia, clinicians must therefore be vigilant of this risk and take a proactive approach to supplementation.

\section{APPROACHES TO PHOSPHATE REPLETION IN CRRT}

Table 1 summarizes the various approaches to phosphate repletion in patients undergoing CRRT, along with their advantages and disadvantages. At present, most centers utilize exogenous supplementation, either oral or parenteral. Supplementation can be a part of a patient's nutritional prescription (e.g. in enteral feeding or total parenteral nutrition), or as dedicated phosphate repletion using intravenous piggyback infusions. In the latter case, phosphate is often administered as part of a hospital's electrolyte protocol. One of the advantages of exogenous supplementation is that the physician has the ability to titrate phosphate to the needs of the clinical situation. However, to avoid hypophosphatemia a high level of vigilance is required, particularly if supplementation is provided by physician order on an ad hoc basis and outside of an established protocol. Even with the use of an electrolyte repletion protocol, there are potential pitfalls. The risk of hypophosphatemia increases when lab draws are infrequent (e.g. once daily), when the threshold level of phosphate for intervention is low, when the amount of supplementation is insufficient, and probably when the route of administration is oral (because of inconsistent absorption). Medical centers have typically developed their own electrolyte supplementation protocols, and at present there are no published consensus protocols.

TABLE 1 Approaches to phosphate supplementation in patients undergoing CRRT

\begin{tabular}{|c|c|c|}
\hline Approach & Advantages & Disadvantages \\
\hline $\begin{array}{l}\text { Physician guided } \\
\text { supplementation }\end{array}$ & $\begin{array}{l}\text { - Use of clinical judgment } \\
\text { - Ease of titration } \\
\text { - Oral/enteral or intravenous options } \\
\text { can be used synergistically }\end{array}$ & $\begin{array}{l}\text { - Relies on high level of vigilance and regular laboratory data } \\
\text { - Vulnerable to solution shortages }\end{array}$ \\
\hline $\begin{array}{l}\text { Protocol-guided } \\
\text { supplementation }\end{array}$ & $\begin{array}{l}\text { - Automated } \\
\text { - Easily titratable supplementation }\end{array}$ & $\begin{array}{l}\text { - May promote less vigilance among clinicians } \\
\text { - Depending on protocol characteristics, may be reactive to } \\
\text { hypophosphatemia instead of proactive } \\
\text { - Vulnerable to solution shortages }\end{array}$ \\
\hline $\begin{array}{l}\text { CRRT solution } \\
\text { supplementation }\end{array}$ & $\begin{array}{l}\text { - Provides steady-state base phosphate level } \\
\text { - Titratable }\end{array}$ & $\begin{array}{l}\text { - Potential risk for errors and/or contamination } \\
\text { - Increased pharmacy workload } \\
\text { - Vulnerable to solution shortages }\end{array}$ \\
\hline $\begin{array}{l}\text { Commercial phosphate-containing } \\
\text { CRRT solution }\end{array}$ & $\begin{array}{l}\text { - Provides steady-state base phosphate level } \\
\text { - No pharmacy manipulation required }\end{array}$ & - Fixed level and nontitratable \\
\hline
\end{tabular}

CRRT, continuous renal replacement therapy. 
In Table 2, we present recommendations for initial phosphate supplementation during CRRT prescribed at standard recommended effluent doses of $20-25 \mathrm{~mL} / \mathrm{kg} / \mathrm{h}$. Because the immediate risks of hypophosphatemia outweigh the risks of mild to moderate hyperphosphatemia, this protocol emphasizes initiation of supplementation at normal to mildly high phosphate levels in order to avoid hypophosphatemia altogether. In contrast, many currently employed protocols focus on repletion only when phosphorus levels have fallen to $2.5 \mathrm{mg} / \mathrm{dL}$ or lower. After initiation, subsequent repletion should be titrated to maintain phosphorus levels in the normal range. Both oral (when patients are tolerating enteral feeding) and intravenous supplementation can be used. Use of low phosphate feeding formulations is not recommended during CRRT. In addition, when phosphate levels are declining, it is important to consider increasing the frequency of monitoring, which allows greater opportunity to avoid overt hypophosphatemia. This recommended protocol is not meant to replace clinical judgment, and clinicians should take into account rate of phosphate change and adjust supplementation accordingly. For example, patients on higher effluent doses of CRRT may require augmented supplementation, while patients with a cell lysis syndrome may not require any supplementation.

The other major approach to prevent hypophosphatemia is to include phosphate in the CRRT solutions. A key advantage to this approach is the ability to maintain a basal level of phosphate that, in theory, patients will not fall below. For example, if CRRT dialysate has a phosphate concentration of $4 \mathrm{mg} / \mathrm{dL}$ ( $1.3 \mathrm{mmol} / \mathrm{L})$, a hyperphosphatemic patient will have a continual clearance phosphate until the serum concentration reaches $4 \mathrm{mg} / \mathrm{dL}$ at which time net

TABLE 2 Recommended protocol for exogenous phosphate supplementation in patients initiating continuous renal replacement therapy using nonphosphate containing solutions

\begin{tabular}{|c|c|c|c|}
\hline $\begin{array}{l}\text { Pre-CRRT } \\
\text { phosphate } \\
\text { level }\end{array}$ & $\begin{array}{l}\text { Oral repletion } \\
\text { regimen }^{\mathrm{a}} \\
\text { (if tolerating } \\
\text { enteral) }\end{array}$ & $\begin{array}{l}\text { IV repletion } \\
\text { regimen }^{\mathrm{b}}\end{array}$ & $\begin{array}{c}\text { Frequency of } \\
\text { phosphorus } \\
\text { monitoring }\end{array}$ \\
\hline$>5.5 \mathrm{mg} / \mathrm{dL}$ & None & None & Q24h \\
\hline $4-5.5 \mathrm{mg} / \mathrm{dL}$ & 1 tab Q12h & None & Q12-24h \\
\hline $2.5-4 \mathrm{mg} / \mathrm{dL}$ & 1 tab Q8h & $\begin{array}{c}\text { None if } \\
\text { tolerating oral. } \\
\text { If unable to } \\
\text { tolerate } \\
\text { oral, then } \\
10 \mathrm{mmol} \text { IV Q12h. }\end{array}$ & Q6-12h \\
\hline$<2.5 \mathrm{mg} / \mathrm{dL}$ & 1 tab Q6h & $\begin{array}{c}\text { 20-40 mmol IV } \\
\text { daily until } \\
\text { normal levels } \\
\text { achieved }\end{array}$ & Q6h \\
\hline
\end{tabular}

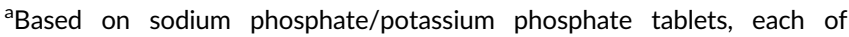
which contains $8 \mathrm{mmol}$ phosphate, $1.1 \mathrm{mEq}$ potassium and $13 \mathrm{mEq}$ sodium. Oral repletion may be reduced if patient is tolerating full diet. bIntravenous phosphate should be given as sodium phosphate unless hypokalemia is also present, in which case potassium phosphate can be used.

CRRT, continuous renal replacement therapy. phosphate clearance will cease. Similarly, a hypophosphatemic CRRT patient would have a continual increase in serum phosphate until their value reaches the $4 \mathrm{mg} / \mathrm{dL}$ equilibrium point. Until recently, centers pursuing this approach have had to add supplemental electrolytes to solutions under sterile conditions to avoid risk of contamination. The availability of commercial phosphate-containing solutions allows for off-the-shelf use of CRRT solutions without additional manipulation; however, the phosphate concentration is fixed in these solutions and is not easily titratable.

\section{5 | OUR SINGLE CENTER EXPERIENCE}

As an internal quality assessment project, we performed a retrospective analysis of 62 consecutive adult patients (36 males and 26 females, mean age $57.5 \pm 11.9$ years) who received CRRT for at least 3 days (mean duration $6.9 \pm 3.5$ days). At the time of this analysis, physicians were administering phosphate as oral or intravenous supplementation, or as an addition to dialysate based on clinical judgment. Figure 1 illustrates that on the first day of CRRT mean initial serum phosphorus concentrations were elevated $6.0 \pm 3.4 \mathrm{mg} /$ $\mathrm{dL}$ but highly variable. Normophosphatemia was achieved in most patients by day 3 , and by day 4 more than half of the patients required some type of phosphate supplementation. The approach chosen most commonly by physicians was the addition of phosphate to the dialysate ( $>50 \%$ of patients).

We observed similar results when examining our experience with pediatric patients undergoing CRRT. Pediatric patients, particularly very young ones, may not have the same phosphate stores as adults and consequently their phosphate supplementation needs in CRRT may differ as well. We compiled the laboratory profiles of 26 consecutive children (mean age $7.3 \pm 7.6$ years) who received CRRT in our institution using a pharmacy-made, phosphate-containing $(2 \mathrm{mmol} / \mathrm{L}$; $6.2 \mathrm{mg} / \mathrm{dL}$ ) dialysate. Soon after this analysis, our institution switched to exclusive use of a commercial, nonphosphate-containing dialysate, to which physicians could order the addition of phosphate. We followed the first 32 pediatric patients receiving this phosphate-free dialysate. They were similar in age to the baseline group ( $9.1 \pm 7.4$ years) and received treatment for $12.8 \pm 13.4$ days. While phosphate control was achieved using both phosphate-containing and phosphate-free dialysate, subjects receiving phosphate-free dialysate were very likely to require the other forms of phosphate supplementation (Figure 2). By day 4 over $90 \%$ of patients starting with the phosphate-free dialysate required added phosphate to the dialysate; over half also were receiving other phosphorus supplementation in the form of oral and/ or intravenous phosphate. In subjects started with the phosphate-containing dialysate, few required extra phosphate in the dialysate and only about a quarter received any intravenous or oral phosphate to maintain desired serum phosphate concentrations. As a result of these observations, our current standard of practice is to routinely add phosphate to our CRRT solutions (target concentration either 0.75 [2.3 $\mathrm{mg} / \mathrm{dL}]$ or $1.5 \mathrm{mmol} / \mathrm{L}$ [ $4.6 \mathrm{mg} / \mathrm{dL}]$ ) in order to limit the risk of hypophosphatemia. 


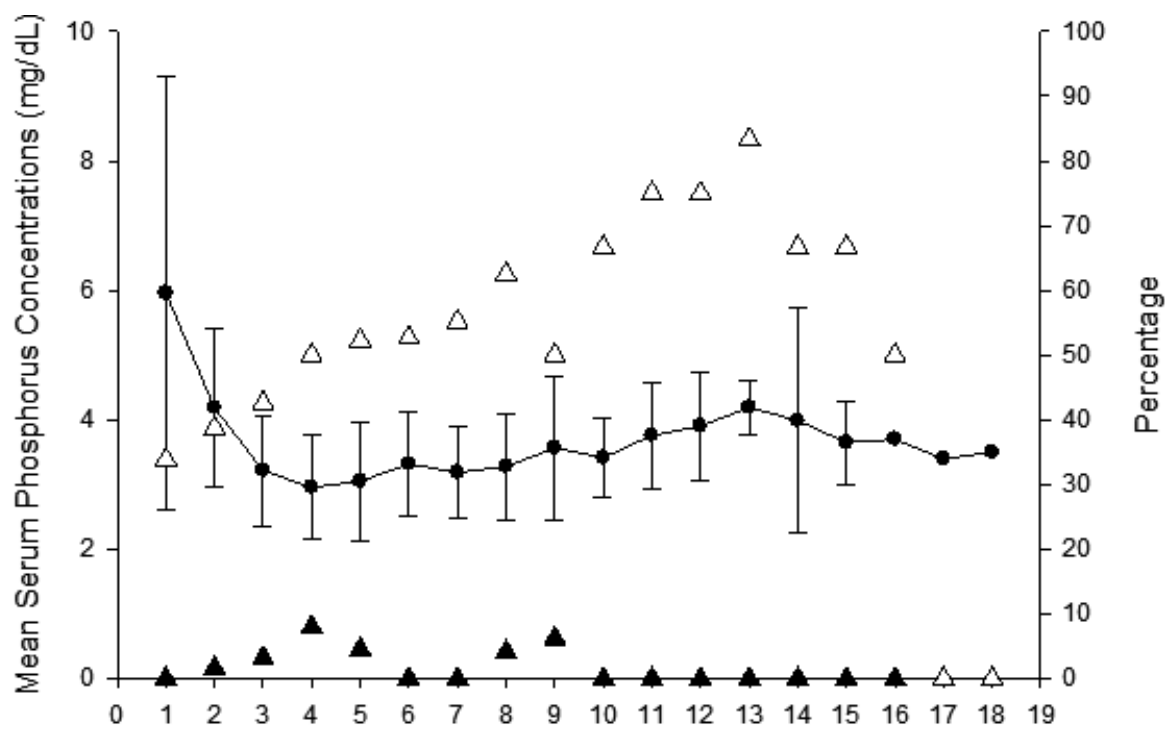

Day of CVVHD Treatment

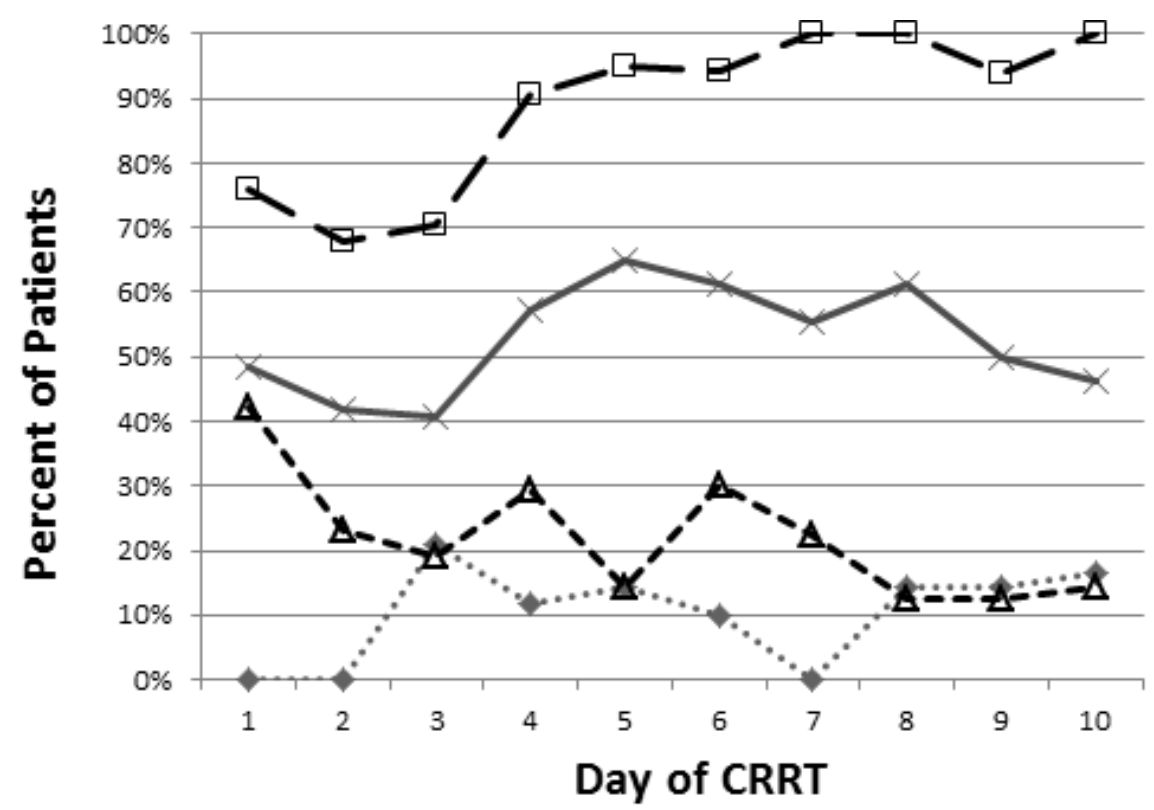

.... Phos added to Phos-containing Dialysate

G Phos added to Phos-free Dialysate

- $\Delta-$ IN/Oral Phos Supplementation in Phos-Dialysate

$\longrightarrow$ IV/Oral Phos Supplementation in Phos-free Dialysate
FIGURE 1 Phosphate supplementation and serum phosphate values in 62 adult patients receiving continuous venovenous hemodialysis. Mean serum phosphate levels denoted by 0 and the error bars on each day denote standard deviation. $\Delta$ denotes percentage of patients who received phosphate into their dialysate. $\Delta$ denotes percent of patients who received exogenous phosphate supplementation either by oral or parenteral routes of administration

FIGURE 2 Daily phosphate supplementation requirements in pediatric patients receiving continuous renal replacement therapy (CRRT) when a phosphate-free dialysate was used $(n=32)$ or a phosphate-containing dialysate was used $(n=26)$. Pediatric patients receiving CRRT with a phosphate-free dialysate required substantially more phosphate supplementation than did those who were receiving a pharmacy-compounded dialysate containing $2 \mathrm{mmol} / \mathrm{L}(6.19 \mathrm{mg} /$ dL) phosphate. By day 4 of CRRT, nearly all patients originally started on phosphatefree dialysate needed to receive phosphate in their dialysate. Intravenous/Oral phosphate supplementation rates were consistently higher for patients receiving phosphate-free dialysate

\section{6 | SPECIAL CONSIDERATIONS FOR PHOSPHATE-CONTAINING CRRT SOLUTIONS}

Several important considerations regarding phosphate supplementation of CRRT solutions are worth noting. First, in recent years there have been shortages of a variety of injectable solutions, including phosphate. ${ }^{6,17}$ Of course, such shortages will also impact exogenous supplementation and may necessitate increased reliance on oral/enteral approaches. During such periods, physicians and pharmacists must work together to identify alternative options and revise approaches as necessary. ${ }^{18}$

Secondly, the United States Pharmacopeia regulations provide guidance on appropriate pharmaceutical compounding practices (USP chapter 797). Appropriately, electrolyte additions to CRRT solutions must be done under controlled sterile conditions and using proper techniques. This requires pharmacy involvement and cannot be done by bedside nursing, so engagement and buy-in from the hospital pharmacy team is critical. Even with proper techniques, contamination remains a theoretical concern. Furthermore, particularly in high- 
volume and high-acuity settings, there is a risk for human error with either incorrect additives or incorrect dosages delivered. Indeed, dialysis solutions are considered "high-alert" medications by the Institute of Safe Medication Practices. ${ }^{19}$ Patients undergoing CRRT are critically ill and vulnerable, and such errors can be devastating. ${ }^{20-22}$

Thirdly, the economic implications of different approaches to phosphate supplementation have not, to our knowledge, been fully explored. In addition to comparing the direct costs (e.g. commercial phosphate-containing solutions versus commercial solutions plus individual additives versus commercial solutions plus supplementation), one must account for the workload associated with custom compounding CRRT solutions. This latter impact will vary by the characteristics of a given medical center, including personnel costs and volume of CRRT care. In our medical center, we have also observed significant medical waste of CRRT solutions which we believe is in part related to a limited shelf life for use (12 hours) after manipulation for electrolyte additives. ${ }^{23}$ Another difficult to quantify consideration is the potential cost from adverse events should they occur.

Lastly, a detailed examination of the recently available phosphate-containing CRRT solution (Phoxillum, Baxter International Inc.) in the United States is warranted. A similar formulation has been available in Europe for a number of years, where published experience suggests efficacy in preventing hypophosphatemia. ${ }^{24,25}$ Studies have also demonstrated the stability of phosphate in CRRT solutions and lack of significant precipitation with calcium. ${ }^{26-29}$ An important difference, however, is that the phosphate concentration of the approved U.S. CRRT solution is $1.0 \mathrm{mmol} / \mathrm{L}(3.1 \mathrm{mg} / \mathrm{dL})$ compared to $1.2 \mathrm{mmol} / \mathrm{L}(3.7 \mathrm{mg} / \mathrm{dL})$ in the European formulation. While this concentration is within the normal range for phosphate, critically ill patients may have higher phosphate needs. This level may also be inadequate for use in pediatric populations who have higher normal phosphate levels. Additional clinical experience with this solution is needed to assess whether exogenous supplementation may still be required. Hospital pharmacy and therapeutics committees will need to assess all these considerations when making their formulary decisions for these new products.

\section{7 | SUMMARY/CONCLUSION}

In summary, hypophosphatemia is a common complication among patients undergoing CRRT and can adversely impact patient outcomes. Unfortunately, hypophosphatemia remains a somewhat overlooked problem, based on the continued high incidence reported in the literature and the fact that most repletion protocols emphasize replacement once hypophosphatemia has developed. A variety of approaches to preventing hypophosphatemia exist, each with their own advantages and disadvantages. Repletion protocols should aim to maintain normophosphatemia and therefore begin supplementation at normal serum phosphorus levels. Based on our experiences, we advocate for addition of phosphate to CRRT solutions in order to prevent development of hypophosphatemia. The recent availability of a commercial phosphate-containing CRRT solution may provide a balance between hypophosphatemia risk, workload and patient safety. However, additional clinical experience with these new solutions is needed.

\section{ACKNOWLEDGMENTS}

The authors thank Brian Church PharmD and Eugene Kreys PharmD for their assistance with clinical data collection.

\section{DISCLOSURES}

Drs. Heung and Mueller have received grant funding from Baxter International Inc. Baxter did not have direct input into the content of this manuscript.

The results/data/figures in this manuscript have not been published elsewhere nor are they under consideration by another publisher.

Michael Heung ${ }^{1}$
Bruce A. Mueller ${ }^{2}$
${ }^{1}$ Division of Nephrology, Department of Medicine, University of
Michigan, Ann Arbor, MI, USA
${ }^{2}$ College of Pharmacy, University of Michigan, Ann Arbor, MI, USA
Correspondence
Michael Heung, Division of Nephrology, Department of Medicine,
University of Michigan, Ann Arbor, MI, USA.
Email: mheung@umich.edu

\section{REFERENCES}

1. Kellum JA, Lameire N, Aspelin P, et al. KDIGO clinical practice guideline for acute kidney injury. Kidney Int. 2012;2:1-138.

2. Ronco C, Bellomo R, Ricci Z. Continuous renal replacement therapy in critically ill patients. Nephrol Dial Transplant. 2001;16(Suppl 5):6772.

3. Demirjian S, Teo BW, Guzman JA, et al. Hypophosphatemia during continuous hemodialysis is associated with prolonged respiratory failure in patients with acute kidney injury. Nephrol Dial Transplant. 2011;26:3508-3514.

4. Yang $\mathrm{Y}$, Zhang $\mathrm{P}$, Cui $\mathrm{Y}$, et al. Hypophosphatemia during continuous veno-venous hemofiltration is associated with mortality in critically ill patients with acute kidney injury. Crit Care. 2013;17:R205.

5. Lim C, Tan HK, Kaushik M. Hypophosphatemia in critically ill patients with acute kidney injury treated with hemodialysis is associated with adverse events. Clin Kidney J. 2017;10:341-347.

6. Hsu RK, McCulloch CE, Dudley RA, Lo LJ, Hsu CY. Temporal changes in incidence of dialysis-requiring AKI. J Am Soc Nephrol. 2013;24:37-42.

7. Kaneko I, Tatsumi S, Segawa H, Miyamoto KI. Control of phosphate balance by the kidney and intestine. Clin Exp Nephrol. 2017;21(Suppl 1):21-26.

8. Felsenfeld AJ, Levine BS, Rodriguez M. Pathophysiology of calcium, phosphorus, and magnesium dysregulation in chronic kidney disease. Semin Dial. 2015;28:564-577.

9. Block GA, Hulbert-Shearon TE, Levin NW, Port FK. Association of serum phosphorus and calcium $\mathrm{x}$ phosphate product with mortality 
risk in chronic hemodialysis patients: a national study. Am J Kidney Dis. 1998;31:607-617.

10. Eloot S, Van Biesen W, Vanholder R. A sad but forgotten truth: the story of slow-moving solutes in fast hemodialysis. Semin Dial. 2012;25:505-509.

11. Spalding EM, Chamney PW, Farrington K. Phosphate kinetics during hemodialysis: evidence for biphasic regulation. Kidney Int. 2002;61:655-667.

12. Kooienga L. Phosphorus balance with daily dialysis. Semin Dial. 2007;20:342-345.

13. Tan HK, Bellomo R, M'Pis DA, Ronco C. Phosphatemic control during acute renal failure: intermittent hemodialysis versus continuous hemodiafiltration. Int J Artif Organs. 2001;24:186-191.

14. Alsumrain MH, Jawad SA, Imran NB, Riar S, DeBari VA, Adelman M. Association of hypophosphatemia with failure-to-wean from mechanical ventilation. Ann Clin Lab Sci. 2010;40:144-148.

15. Kilic O, Demirkol D, Ucsel R, Citak A, Karabocuoglu M. Hypophosphatemia and its clinical implications in critically ill children: a retrospective study. J Crit Care. 2012;27:474-479.

16. Bellomo R, Cass A, Cole $L$, et al. The relationship between hypophosphataemia and outcomes during low-intensity and high-intensity continuous renal replacement therapy. Crit Care Resusc. 2014;16:34-41.

17. Lemon SJ, Zack SD, Voils SA. No difference in mechanical ventilation-free hours in critically ill patients who received intravenous, oral, or enteral phosphate replacement. J Crit Care. 2017;39:31-35.

18. Holcombe B, Andris DA, Brooks G, Houston DR, Plogsted SW. Parenteral nutrition electrolyte/mineral product shortage considerations. JPEN J Parenter Enteral Nutr. 2011;35:434-436.

19. Wilson FP, Yang W, Feldman HI. Predictors of death and dialysis in severe AKI: the UPHS-AKI cohort. Clin J Am Soc Nephrol. 2013;8:527-537.

20. Barletta JF, Barletta GM, Brophy PD, Maxvold NJ, Hackbarth RM, Bunchman TE. Medication errors and patient complications with continuous renal replacement therapy. Pediatr Nephrol. 2006;21:842845.

21. Johnston RV, Boiteau P, Charlebois K, Long S, U D. Responding to tragic error: lessons from Foothills Medical Centre. CMAJ. 2004;170:1659-1660.

22. Pendergrast JM, Hladunewich MA, Richardson RM. Hemolysis due to inadvertent hemodialysis against distilled water: perils of bedside dialysate preparation. Crit Care Med. 2006;34:2666-2673.

23. Benfield $C B$, Brummond $P$, Lucarotti $A$, et al. Applying lean principles to continuous renal replacement therapy processes. Am J Health Syst Pharm. 2015;72:218-223.

24. Chua HR, Schneider AG, Baldwin I, Collins A, Ho L, Bellomo R. Phoxilium vs Hemosol-BO for continuous renal replacement therapy in acute kidney injury. J Crit Care. 2013;28:884.e887-814.

25. Morabito S, Pistolesi V, Tritapepe L, et al. Continuous veno-venous hemofiltration using a phosphate-containing replacement fluid in the setting of regional citrate anticoagulation. Int $J$ Artif Organs. 2013;36:845-852.

26. Troyanov S, Geadah D, Ghannoum M, Cardinal J, Leblanc M. Phosphate addition to hemodiafiltration solutions during continuous renal replacement therapy. Intensive Care Med. 2004;30:1662-1665.

27. Santiago MJ, Lopez-Herce J, Munoz R, et al. Stability of continuous renal replacement therapy solutions after phosphate addition: an experimental study. Ther Apher Dial. 2011;15:75-80.

28. Besnard N, Serveaux M, Machado S, et al. Electrolytes-enriched hemodiafiltration solutions for continuous renal replacement therapy in acute kidney injury: a crossover study. Blood Purif. 2016;42:18-26.

29. Broman M, Carlsson O, Friberg H, Wieslander A, Godaly G. Phosphate-containing dialysis solution prevents hypophosphatemia during continuous renal replacement therapy. Acta Anaesthesiol Scand. 2011;55:39-45. 